\title{
Decomposition (Ferrogravitolysis) Water and Hydrocarbons by means of Technical Ferrogravitational Field
}

\author{
Robert A. Sizov
}

\begin{abstract}
Experimental and theoretical studies of the author (period: 1968 - present) have shown that true sources of magnetic field are magnetic fundamental particles (magnetic charges), and not moving electrons. The main reason for the vicious ignorance of magnetic charges in physics is the super-hard conditions of confinement of these particles in atoms and substance which are cardinally different from the confinement of electrons. Magnetic charges together with electric charges form the shells of atoms which in reality are electromagnetic, and not electronic. The orbital electromagnetic currents in atomic shells are sources of the gravitational field which is the vortex electromagnetic field and is described by vortex vector $\operatorname{rot}[\mathbf{E}-\mathbf{H}]$. Depending on the state polarization of vortex vectors $\operatorname{rot}[\mathrm{E}-\mathrm{H}]$ in compositions of the atomic gravitational fields its subdivided into paragravitational (PGF) and ferrogravitational fields (FGF). The main process defining formation of chemical compounds is the gravito-chemical binding or compression of atoms by the forces of the paragravitational "Dark Energy" which is realized between PGF atoms-sources. Between the atoms emitting PGF and FGF the effect of Gravitational levitation is realized the forces of which push the atoms away from each other. Therefore, the combination of carbon atoms (PGF sources) and hydrogen atoms (FGF-sources) with the formation of hydrocarbon molecules can be realized only if the hydrogen atoms are in molecular form $\left({ }^{1} \mathrm{H}_{2}\right)$. In the composition of water, within the framework of the chemical formula $\mathrm{H}_{2} \mathrm{O}$, two stable isomorphic molecular structures are realized, the gravito-chemical bond in which is carried out, respectively of the hydrocarbon scenario described above, as well as under conditions of unidirectional ferropolarization of the gravitational fields of atoms in molecule and of the implementation of the so-called hydrogen bond. The external (technical) ferrogravitational field violates the initial (paragravitational) state of gravitational fields in interatomic regions what leads to the formation of positive (gravitational) "Dark energy" the forces of which break interatomic chemical bonds and leads to molecular decays. This process, by analogy with process of electrolysis, can be referred to as ferrogravitolysis. The Author's technology for the decomposition of water molecules by means of FGF is a technical analogue of the natural process of water decomposition which is realized in the cells of leaves of green plants and algae. The intensity of technical FGF, and, consequently, the efficiency of the process of decomposition water molecules can be thousands of times higher than the efficiency of its natural decomposition in plant cells, which makes it possible to obtain cheap green hydrogen for wide practical use. In addition, the effectiveness of the impact of (technical) FGF on the state of gravitational fields in the interatomic regions of molecules and the implementation of the process of their decomposition is many times greater than the capabilities of the currently used electric field.
\end{abstract}

Keywords - "Dark energy", ferrogravitolysis, Ferro-and paragravitation, Gravitational field, Gravitational levitation, Gravito-chemical bond, Magnetic charges, Magnetic dipoles, S-Gravitons, true Antielectrons.

\section{Real Magnetic Charges in Structures of Atoms AND Substance, ParA- AND ERROGRAVITATION, GRAVITATONAL LEVITATION}

The experimental and theoretical studies carried out by the author (period: 1968 - present) have shown that the magnetic fundamental particles (magnetic charges) are real structural components of atoms and substance and are the immediate sources of all magnetic fields in Nature [1]-[5]. Magnetic particles with negative charge $\left(\mathrm{g}^{-}\right)$existing in the compositions of atomic shells received author's name magnetons, that should not be confused with such the theoretical surrogates as the Bohr's magnetons. Magnetons in its physical parameters are magnetic analogs of electrons. Together with electrons and magnetons in compositions of atomic shells there are their antiparticles, i.e., true antielectrons $\left(\mathrm{e}^{+}\right)$and antimagnetons $\left(\mathrm{g}^{+}\right)$. Thus, atomic shells in reality are electromagnetic and not electronic, as it is believed, and a magnitude charges of electric and magnetic particles in atomic shells correspond to the condition $\mathrm{e}=\mathrm{g}$ [6]. In addition

Submitted on November 03, 2021

Published on December 13, 2021.

Robert A. Sizov

(corresponding e-mail: sizov.robert@gmail.com) 
to participating in atomic structures magnetic charges, together with electric charges, populate potential conduction bands in conductors, which, under superconductivity conditions, can transform for them into zones of real magnetic conductivity [7].

The true sources of magnetic field, i.e., real magnetic charges turned out to be "buried alive" in physical science due to the conditions of their hard confinement in atoms and substance which are radical different from the confinement of electrons. Along with magnetic charges under conditions of rigid confinement in substance there are and such fundamental particles as true electrons, which are also not recognized in the existing physics [8].

Very negative role in the tragic history with real magnetic charges played the vicious Maxwell's concept of electric magnetism (1873) which is the result of the superficial and, exclusively erroneous, impression of the Great Physicist from the famous experience of Oersted [9], [10]. The detailed analysis of the reasons that led to the vicious ignoring, for almost 150 years, of magnetic charges and true antielectrons in existing physics are given in the Appendix to the article.

It is important to emphasize that the above-mentioned real magnetic poles (magnetic charges) have nothing in common with the known Dirac monopoles [11], [12], and also do not participate in such physical manifestations as the magnetricity [13]. All these last theoretical constructions and manifestations in the formation of magnetic field used electrons and their currents, i.e., they are, in fact, of electrified constructions based on Maxwell's vicious electric magnetism [14].

The joint orbital currents of electric and magnetic charges in atomic shells are the source of the gravitational field (GF) which is the vortex electromagnetic field. The elementary source of gravitational field is the vortex electromagnetic quasi-particle which received the name $\mathbf{S}$-Graviton ( $\mathrm{S}=$ source). The S-Graviton is combination of the electric and magnetic dipoles rotating in antiphase at same atomic orbital. The model representation of the orbital electromagnetic current or S-Graviton can be written in following form: $\operatorname{rot}\left[\mathbf{J}_{\mathrm{e}}-\mathbf{J}_{\mathrm{g}}\right]$, where $\mathbf{J}_{\mathrm{e}}$ and $\mathbf{J}_{\mathrm{g}}$ are vectors the density instantaneous orbital currents of electric (e) and magnetic $(\mathrm{g})$ charges. Then the equation process gravitational field formation by means the S-Graviton can be presented in the form:

$$
\mathrm{k} \operatorname{rot}\left[\mathbf{J}_{\mathrm{e}}-\mathbf{J}_{\mathrm{g}}\right]=\operatorname{rot}[\mathbf{E}-\mathbf{H}]
$$

where $\mathbf{E}$ and $\mathbf{H}$ are the vectors of the instantaneous electric and magnetic field strength in the structure of the vortex electromagnetic (gravitational) field, $\mathrm{k}$ is the proportionality coefficient. The minus signs indicated in the above equation of formation gravitational field correspond to both the antiphase of the orbital currents of electric and magnetic charges, so and the anti-directionality of the vectors of the instantaneous electric and magnetic field strength at each point of gravitational field.

Vector-vortex analogy between magnetic (a) and gravitational (b) of fields is shows on Fig. 1. Unlike from vortex magnetic field of every point which is answered one vector of instantaneous strength of $\mathbf{H}$, every point of gravitational field is responsible two vectors of instantaneous strength of fields $\mathbf{E}$ and $\mathbf{H}$ equal by value and oriented antiparallel to each other. In addition, in Fig. 1 shown that the magnetic vortex field, which is determined by the vortex vector rotH is formed by means of the rotating the magnetic dipole. The elementary source gravitational field is S-Graviton which can be defined as the dynamic EM-bidipole, i.e., two related dipoles (electric and magnetic) rotating in opposite phase on one atomic orbit. The classical equations of the processes formation magnetic and electromagnetic (gravitational) fields also shows in Fig. 1.

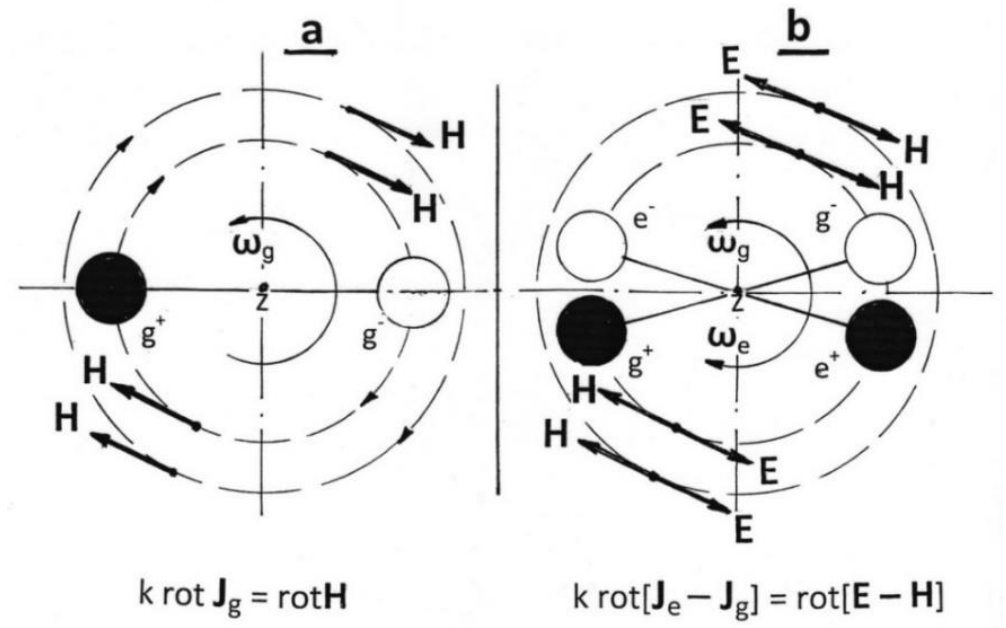

Fig. 1. Schemes of mechanisms formation of the vortex spinor fields: magnetic (a) and electromagnetic (gravitational) (b). By white circles on the Fig. 1 showed negative charged electric and magnetic spinors: electron $\left(\mathrm{e}^{-}\right)$and magneton $\left(\mathrm{g}^{-}\right)$and black circles is positively charged antispinors corresponding to them with charges $\mathrm{e}^{+}$and $\mathrm{g}^{+}$. 
If polarization of vortex vectors $\operatorname{rot}\left[\mathbf{J}_{\mathrm{e}}-\mathbf{J}_{\mathrm{g}}\right]$ of $\mathrm{S}$-Gravitons is realized in the structures of Physical masses (in atoms, nucleons, substance et al) that is accompanied by polarization of vortex vectors rot[E $\mathbf{H}$ ], then by analogy with magnetic fields of ferromagnetics the gravitational fields being emitted these masses can be called the ferrogravitational fields (FGF). The gravitational fields formed by the physical masses in the absence polarization of the vortex vectors of S-gravitons in their structures are tensor or quasiscalar fields. And again, by outward analogy with magnetism such fields can to define as paragravitational fields (PGF). It is important to note that the system of two coupled of the electric and magnetic dipole rotating in antiphase in one atomic orbit is real shell of a hydrogen atom (protium).

The mathematical expression corresponding to the state of FGF is have the form $<\operatorname{rot}[\mathbf{E}-\mathbf{H}]>\neq 0$. The gravitational field corresponding to condition $\langle\operatorname{rot}[\mathbf{E}-\mathbf{H}]\rangle=0$ by analogy with the paramagnetism can be defined as paragravitational field (PGF).

As you know, the magnetic vortex field is determined at each point by one intensity vector $\mathbf{H}$. As for the gravitational field, it is determined at each point by two intensity vectors $\mathbf{E}$ and $\mathbf{H}$ which are equal in magnitude and oppositely directed relative to each other (see Fig. 1(b)). Thus, GF can be defined as an electromagnetic vortex bivector field, in which the vectors of the strength of the electric $\mathbf{E}$ and magnetic $\mathbf{H}$ fields make up a single electromagnetic bivector $(\mathbf{B V})$, described as $[\mathbf{E}-\mathbf{H}]$ or, simply, $\mathbf{E}-\mathbf{H}$. According to the noted vector conditions, we will further to determine the directionality of the FGF both through the vector of its ferropolarization $\mathbf{P}_{\mathrm{fp}}$ and through its bivector $\mathbf{B V}$.

As part of the experimental detection and study of real magnetic charges, the author has implemented a method for separating magnetic charges into pairs $\mathrm{g}^{-}$and $\mathrm{g}^{+}$, and also developed a technology for enriching the obtained magnetic charge. Using the obtained difference of magnetic charged potentials, he realized currents of magnetic charges in a lead superconductor. The methodology and results of the author's experiments with real magnetic charges and their currents are described in detail in the author's book (cm. [5]), and are also presented in articles [1]-[4].

Note 1. It is important to note that much earlier than the author, i.e., in 1910-1952, magnetic charges on particles of various substances were observed by the remarkable Austrian physicist Felix Ehrenhaft during very delicate and laborious experiments [15]. Ehrenhaft's experiments were repeated by his numerous followers who also came to the conclusion about the existence of real magnetic charges (see, for example, [16]). At the same time, according to the results of the above experiments, only a statement of the existence of magnetic charges was made. As for the physics of magnetic particles, as well as their place in the structures of substance, all this remained unknown. This is, most likely, the main reason that Ehrenhaft's magnetic charges were not perceived by the physical community and are now practically forgotten.

\section{The World PhySicAl TRIAD, SPINOR FIELDS AND “DARK ENERGY” IN PROCESSES OF THE FORMATION AND DECOMPOSITION OF MOLECULES}

The results of years of research of the real magnetic spinor particles and introduction it to basic physics allowed the author to formulate the conception of the World Physical Triad (WPT) according to which the real World consists of three fundamental phases: Matter, Antimatter and Energo-phase (Energomedium) [17], [18]. The Matter or Material phase is formed by electric and magnetic particles with negative charge ( $\mathrm{e}^{-}$and $\mathrm{g}^{-}$) which defined as spinors. As for the antimaterial phase it is formed by antspinors, i.e., electric and magnetic fundamental particles with a positive charge $\left(\mathrm{e}^{+}\right.$and $\left.\mathrm{g}^{+}\right)$. Particles of the Antimater phase constitute half all of real spinor particles, i.e. charged particles in real World, and their absence in the physical representations is determined by Physics of hard confinement these particles in structures of substance. The process so-called annihilation of pair: spinor and antispinor, is accompanied by a strong compression of these particles to one another by forces "Dark Energy" ("DE") and, in principle, not lead to the annihilation of the particles.

Note 2. In Physics with real magnetic charges the concept of Matter or Material phase refers exclusively to fundamental electric and magnetic particles with a negative charge. A representative of Matter is, for example, an electron. As for positively charged fundamental particles, both electric and magnetic, they constitute Antimatter or Antimaterial phase, which should not be confused with such a concept as antisubstance. The representative of the Antimaterial phase is the true antielectron $\left(\mathrm{e}^{+}\right)$which together with magnetic charges is not recognized in physical science (see, [18]).

The Energo-phase (Energo-medium, Power-medium) is of the global medium which executes all force acts on the particles and masses in real World. In the basic (undisturbed) state the Energo-phase is the isotropic superhigh-density gas-like (maybe even quasi-fluid) medium formed by its own fundamental particles referred to as the energions (denoted by the letter " $\varepsilon$ ") which are spinless and massless. These particles are very small, they move in all directions at speeds close to the speed of light and can be only the lefts and rights, what linked with the appropriate direction of their own rotation. General state of the energions within Energo-medium is determined as doubly degenerate. Super-high mobility and not-inertial 
behavior of the energions allow the particles and masses to move relatively freely in Energo-phase when this medium is in basic (undisturbed) state.

Note 3. The Energo-medium can also be called as the Energo-ether, and instead of the ethereal particles known as the Amers in composition of the Physical Triad were been injected particles of the energions. The introduction of the prefix "Energo" in the composition of the name third component of WPT and its particles is a reflection of the global force significance of this phase in the dynamics of particles and bodies in the real World.

According to the Physical Triad Concept all forces of direct action on the particles and masses which are implemented in the Energo-phase are the forces of the so-called "Dark Energy" ("DE") which is determined by non-equilibrium states in Energo-medium in the form of oblasts of local pressures created by its own particles - energions. It is the "Dark energy" induced in Energo-medium by spinor fields are the fundamental source of all known force manifestations in nature. Therefore, the "DE" forces in the "Energomedium" are responsible for the processes of condensation (binding) of particles of Material and Antimaterial phases into electromagnetic condensates called Physical Mass (FM). Physical masses are: atoms, nucleons, substance and others. The fundamental property of FM is the ability to emit a gravitational field. In other words, everything that can emit a gravitational field is the Physical Mass. Recall that in Physics with real magnetic charges the gravitational field is a vortex electromagnetic field which is described by vortex vector $\operatorname{rot}[\mathbf{E}-\mathbf{H}]$.

Fig. 2 presents a scheme of the World Physical Triad which includes three fundamental World phases: Matter, Antimatter and Energo-medium (Energo-phase). The fundamental particles that constitute Phases of the Physical Triad are indicated on the diagram, i.e. the spinors, antispinors and energions. In Fig. 2 marked and such derivatives from phases of Triad as the spinor fields and "Dark energy". Outside of this scheme remain are the condensates consisting of the spinor particles of Matter and Antimatter, called Masses.

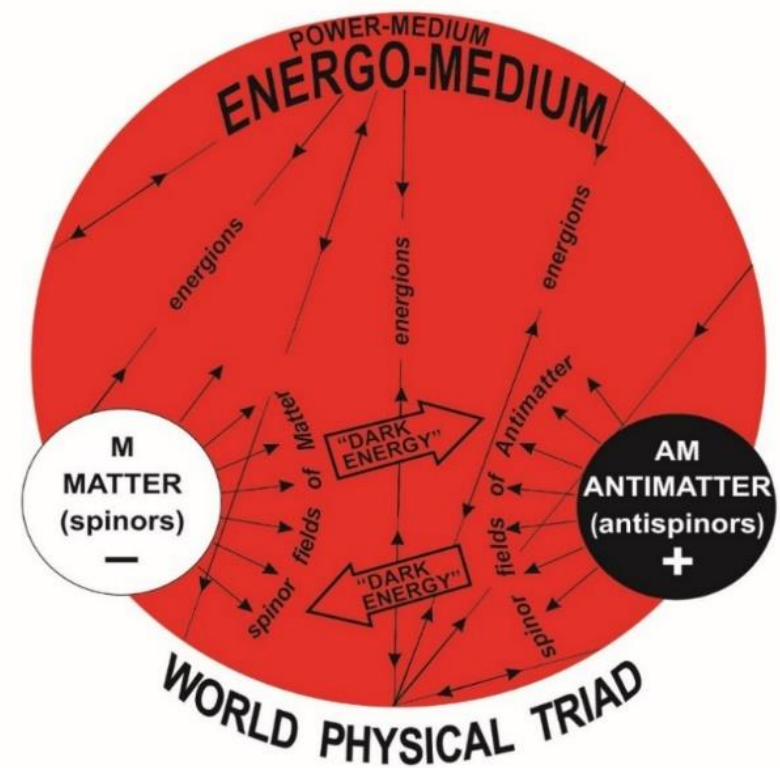

Fig. 2. The Diagram of the World Physical Triad.

The energions, i.e. the own particles of the Energo-medium are the filling of the spinor fields i.e., the charged particle fields. Depending on the direction of rotation of energions in the flow of the spinor field these fields are subdivided into left-handed (the electric fields) and right-handed (magnetic fields). Spinor fields create dissipation of energy in the Energo-medium that and manifests itself in the form of areas positive pressure (positive "DE") and areas with negative pressure (negative "DE") relative to the average pressure $<\mathrm{P} \varepsilon>$ in its of the regional space.

Note 4. The term "spinor fields", if we strictly approach this definition, refers to the fields or fluxes of particles of energions that are formed in the Energo-medium by means of spinors, i.e. particles with negative magnetic and electric charges. As for the antispinors, i.e. particles with a positive charge, then their fields are anti-fluxes of energions and such fields should be referred to as anti-spinor fields. At the same time, taking into account that spinor fields in physics are the primary manifestation in relation to anti-spinor fields, as well as the fact that in the physics under consideration these fields are in natural unification, we, in what follows, will refer to all fields of charged particles will be referred to as as spinor fields (see [18]).

The well-known fundamental forces: gravitational, electromagnetic, strong and weak, are nothing but the manifestation of "DE" formations in the form of pressures $\mathrm{P} \varepsilon$ in the Energy medium induced by the 
corresponding spinor fields. The above statement is not difficult to accept with respect to the first two forces, which are determined respectively by the gravitational "DE" and electromagnetic "DE". As for the so-called strong and weak interactions, as shown in [19], the forces corresponding to them are also the result of "DE" induced by the corresponding spinor fields.

Thereby all of spinor fields, including gravitational fields, don't have real of power significance. They only play the role of intermediaries exerting influence on state of the Energo-medium and inducing formation of "Dark Energy" in it. Namely "Dark Energy" is real source of forces which are responsible for the dynamics of particles and bodies, as in the scale of the Universe (the movement of galaxies, stars, planets and other objects) so and in the microcosm, for example, in chemical bond formation and dynamics of the spinor particles within in atoms, nucleons, etc.

Between the masses (bodies) that emit paragravitational field is implemented generally accepted in of physical science the attraction or gravity. Since the overwhelming number of masses in the Universe emit the paragravitational field the processes of "attraction" between them define the famous Law of universal gravitation. In physics with real magnetic charges, developed by the author, it is shown that behind the processes of the so-called attractions, in fact, there are processes of pressing masses to each other by the forces "Dark Energy" ("DE") and, mainly, by forces of paragravitational "DE". A classic example of the latter process is the gravitational pressing of the so-called paragravitational masses to the paragravitational Earth, which occurs in reality and is described by the law of universal gravitation.

The masses which emit FGF repelled from is masses-sources the PGF, for example, from Earth. This last effect, discovered by the author of the article, called the effect of Gravitational Levitation (GL) [20].

For the purposes of this article, it is important to note that atoms, depending on physical conditions, can emit both PGF and FGF. Between the PGF and FGF source-atoms, forces of positive "DE" are realized, which repel atoms from each other, just as the Earth's para-gravitational field pushes out the sources of the ferrogravity field, for example, light hydrogen (protium) atoms. It is the noted effect and the forces of positive "DE" realized between atoms-source of the PGF and FGF determine the physics of the decomposition processes of hydrocarbon and water molecules in the author's technology presented in the article.

\section{Electromagnetic SHElls OF Atoms, GRAVITO-CHEMistRy AND A Viciousness OF THE THEORY ELECTRONIC CHEMICAL BOND}

The discovery and introduction into the basic physics of real magnetic charges, as well as true antielectrons which together with electrons make up the shells of atoms allowed the author to develop and propose new vision of the nature of chemical bonding which is based the concept of the gravito-chemical bonding [21]. The forces responsible for this chemical bond are the result of the formation of negative "DE" in the interatomic regions under the influence of paragravitational field. It is the paragravity state (PG-state) of GF in interatomic regions which are initiated by atomic gravitational fields are the main reason for the realization of all acts of gravito-chemical bonding and the formation of an overwhelming number of chemical compounds.

The exception here is the ionic bond in ionic crystals. However, all ions have electromagnetic shells and generate a gravitational field. Therefore, ionic bonding is relatively rare addition to the processes of gravitochemical bond. In addition, it is important to note that the existing concept of ion bonding arose on the basis of vicious model purely electronic shells of atoms and in the absence of ideas about the real physics of the gravitational field. For example, the version of ion binding in water and hydrocarbon molecules is, according to the author, erroneous. Of course, the hydrogen atom (protium) carries a positive electric charge $\mathrm{e}^{+}$, but this charge is determined by the proton of its nucleus and has nothing to do with the ionic state of the atom.

The formation of chemical compounds, at the moment, is described on the basis of the theory of electronic chemical bonding which arose as the result of vicious situation in the natural sciences when physicists have "bury alive" the real magnetic charges, as well as true anti-electrons and announced that the shells of atoms are purely electronic.

In the described situation which has developed in of the physical-chemical science, the vicious concept of electronic chemical bonding turned out to be the only "life-saving device" using which it was possible all to at least somehow explain. You don't need to be the great thinker to see all the grandiosity and, at the same time, the impracticability of the task that faced the creators and users of the theory of electronic chemical bonding. The problem is that this theory had to explain the complex physic-chemical processes occurring in atomic condensates, using for this only the electron, that is, one of the four real elementary particles that make up the atomic shells.

However, it was impossible to completely get rid of magnetic charges and true anti-electrons in physical theory since their physical "ears" invariably was "stick up" in the results of experiments. Over time, 
magnetic charges in physical theory were replaced by such theoretical surrogates as the magnetic moments of electrons, and true antielectrons by Dirac holes or electron vacancies [22]. It should be noted here that this last theoretical "trick" not only did not resolve the vicious situation in physics, but also created additional super-difficulties at interpreting the effects and manifestations put forward by Nature.

\section{The Ferrogravitolysis Hydrogen, Hydrocarbons AND WATER MOLECULES}

\section{A. The Hydrogen Gravito-chemical Bond and Ferrogravitolysis of Molecule ${ }^{l} \mathrm{H}_{2}$}

The author's studies have shown that the main mechanism of chemical bonding in nature is the gravitochemical bond (GCB) which is the result of a formation of PGF in interatomic regions. It is the last fields that initiates the negative "DE", the forces of which bonding the atoms into the compositions of chemical compounds.

Let us note here as very important: the states of para-ferro of gravitational field in the interatomic regions correspond to the physical processes the bonding and repulsion of atoms in molecules.

Of course, the formation of PGF in interatomic regions and the implementation of the gravito-chemical bond is carried out, as a rule, when participation the so-called of paragravitational atoms. However, there is a possibility of GCB formation and between the atoms emitting FGF. Such a bond is realized, for example, between hydrogen atoms (protium), which are stable sources of FGF and underlie the synthesis of ${ }^{1} \mathrm{H}_{2}$ molecule. The essence of the physics of this chemical bond can be understood from Fig. 3, which shows two hydrogen atoms (protium), the ferropolarization vectors $\mathbf{P}_{\mathrm{fp}}$ of the gravitational fields of which are unidirectional. The dotted concentric arrowed lines in Fig. 3 show the FGF emitted by hydrogen atoms.

From Figure 3 it is easy to see that the PGF region is realized between the hydrogen atoms which initiates the formation of a negative "DE" here. It is the forces $\mathbf{F}$ this "DE" that are responsible for the interatomic bond in the ${ }^{1} \mathrm{H}_{2}$ molecule, which can be called the hydrogen bond. The distribution of pressures $\mathrm{P}_{\varepsilon}$ of "DE" in the Energo- medium, the forces of which determine the GCB in the hydrogen molecule, is shown in the lower part of Fig. 3.

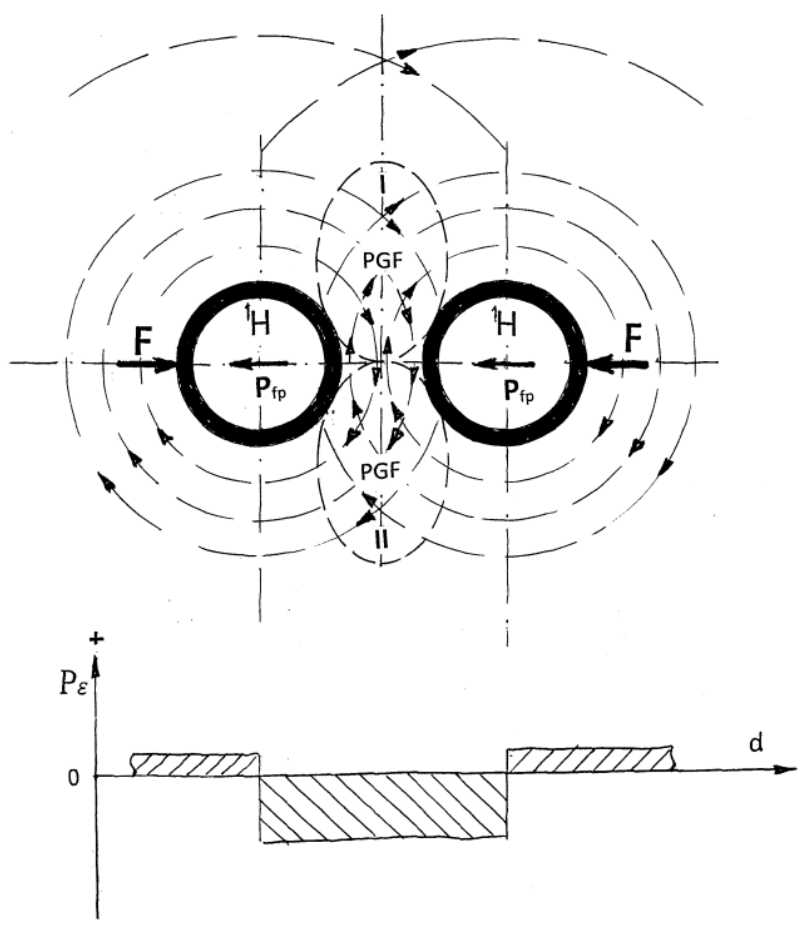

Fig. 3. Diagram of the device, gravitational fields of atoms and forces of gravito-chemical bond in the hydrogen molecule ${ }^{1} \mathrm{H}_{2}$.

Dotted strokes in Fig. 3 shows two eleptic regions I and II in the composition of molecular GF, in which PGF prevails. Below in the article it will be shown that it is these areas that are responsible for the gravitochemical bond of the hydrogen molecule in the composition of hydrocarbon and water molecules.

Thus, the conditions of the chemical bond, which is realized between the ${ }^{1} \mathrm{H}$ atoms emitting FGF (see Fig. 3), ultimately comes down to the formation of a paragravitational field region between them, which can be referred to as molecular PGF.

It is important to emphasize here again that the formation of PGF and, consequently, of negative "DE" between atoms, under conditions of hydrogen bonding, is determined by the conditions of co-directivity vectors of the vectors $\mathbf{P}_{\mathrm{fp}}$ of atomic $\mathrm{FGF}_{\mathrm{s}}$. As noted above, in addition to the ferropolarization vectors of 
$\mathrm{FGF}_{\mathrm{s}}$, the direction of such fields can also be determined by means of their bivector (BV) of intensity $\mathbf{E}-$ $\mathbf{H}$, which we will do in relation to the external (technical) FGF.

The physics of gravity-chemical bond discovered by the author and presented in his articles, suggests that for the decomposition of chemical compounds it is necessary to disrupt the paragravitational state of $\mathrm{GF}_{\mathrm{s}}$ in interatomic regions, i.e. realize their the ferropolarization. It is easy to see that the ferrogravitational field is here an organic destroyer of the paragravitational state and its use is the most effective way of decomposing molecules from all possible ones.

Note 5. In full accordance with the meaning of the word "electrolysis", i.e., "decomposition (disintegration) of a substance into its constituent parts during the passage of an electric current", in our articles we will use such a definition as "ferrogravitolysis", i.e. decomposition (disintegration) of a substance (molecules) under the influence of the technical ferrogravitational field.

It is also important to noted that the technology described in article is a technical analogue of the natural process of decomposition of $\mathrm{CO}_{2}$ and $\mathrm{H}_{2} \mathrm{O}$ molecules by FGF i.e. of the ferrogravitolysis, in cells of leaves of green plants and algae (see Section 5 of this article).

Fig. 4 shows two schemes of physical impacts of external FGF (shown by solid concentric lines) on the ${ }^{1} \mathrm{H}_{2}$ molecule, which differ only in the orientation of the bivector or $\mathbf{E}-\mathbf{H}$ of the technical FGF relative to the axis of the hydrogen molecule. It is important to note that the bivectors FGF of hydrogen atoms, within the framework of the presented processes of decomposition of the molecule, similar to the conditions of magnetization, are oriented along the bivector of the technical field.

Fig. 4a shows diagram of a process in which the $\mathbf{B V}$ of the outer FGF is directed normal to the molecule axis (the lines of this field are shown in Fig. $4 \mathrm{a}$ by solid concentric lines with arrows). It can be seen that in this geometry the external FGF does not affect the vector state of GF in the interatomic region of the molecule, which remains PGF (marked by condition $\left\langle\mathbf{P}_{\mathrm{fp}}\right\rangle=0$ ).

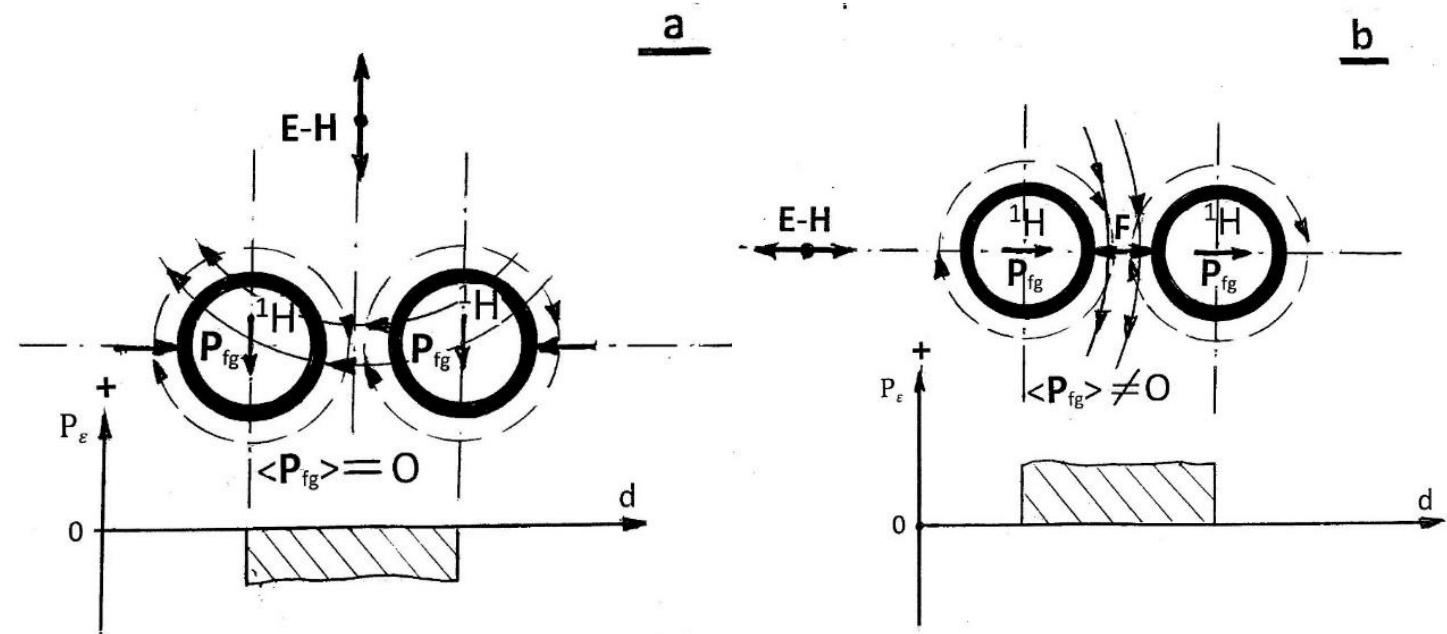

Fig. 4. Schemes of physical impacts of external FGF (shown by solid concentric lines) on the ${ }^{1} \mathrm{H}_{2}$ molecule: which differ only in the orientation of the bivector or E-H of the technical FGF relative to the axis of the hydrogen molecule.

The situation changes dramatically in the case of the directionality $\mathbf{B V}$ of the technical FGF along the axis of the molecule (Fig. 4b). In this case, a ferrogravitational component is realized in the interatomic region, which converts the original PGF into FGF. In this case, a region of positive "DE" is realized between the atoms, the forces of which push away the atoms in the molecule apart. The magnitude of these forces depends both on the strength of the technical field and on the orientation of its $\mathbf{B V}$ relative to the axis of the molecule.

The lower part of Fig. $4 \mathrm{a}$ and $4 \mathrm{~b}$ shows the diagrams of the pressure distribution $\mathrm{P} \varepsilon$ in the Energy medium, which determine the formation of the "DE" forces that act on the atoms in each of the above options of impact.

With regard to the latter processes, it is important to note that the existing representations of ionic states and interactions during the formation of hydrocarbon and water molecules do not correspond to reality, which is determined by the use of the vicious concept of electronic chemical bond and of appropriate physical interpretations.

The noted forces of positive "DE", described and leading to the disintegration of the molecule, if you do not delve into the details of the real physics of this process, can be defined as entropic forces corresponding to the so-called entropic interaction.

\section{B. The Structural Device and Ferrogravitolysis of Molecules Hydrocarbons}

The basis of the chemical bonding of atoms in the composition of chemical compounds, according to the 
results of the author's research, is the gravito-chemical bond, which is carried out by the "DE" forces, initiated in the interatomic regions by paragravitational fields. Thus, to implement the decomposition processes of compounds, it is necessary to destroy the para-gravitational state of $\mathrm{GF}_{\mathrm{s}}$ in the interatomic regions and transfer it to the ferrogravitational state [24]. All of the above, in full measure, also applies to the molecules of hydrocarbons and water.

The structural device of hydrocarbon molecules of the alkane class, within the framework of the concept of gravito-chemical bond (GCB), was studied in detail by the author and presented in his publication [25]. As shown above in Section 4.1 (see Fig. 3), two local regions of the paragravitational field are realize around the ${ }^{1} \mathrm{H}_{2}$ molecule which make it possible to exclude gravito-levitation repulsion between the carbon atom and the hydrogen molecule and to form hydrocarbon molecules (the marked regions with predominant PGF are designated as I and II).

The detailed chemical formulas of the simplest hydrocarbon molecules that meet the conditions of gravito-chemical bond, as well as their structural motifs in chains view, have the following form: $\mathrm{H}_{2} \mathrm{CH}_{2}$ and $\mathrm{H}_{2}=\mathrm{C}=\mathrm{H}_{2}$ (methane), $\mathrm{H}_{2} \mathrm{CH}_{2} \mathrm{CH}_{2}$ and $\mathrm{H}_{2}=\mathrm{C}=\mathrm{H}_{2}=\mathrm{C}=\mathrm{H}_{2}$ (ethane), $\mathrm{H}_{2} \mathrm{CH}_{2} \mathrm{CH}_{2} \mathrm{CH}_{2}$ and $\mathrm{H}_{2}=\mathrm{C}=\mathrm{H}_{2}=\mathrm{C}=\mathrm{H}_{2}=\mathrm{C}=\mathrm{H}_{2}$ (propane).

The structural device of other hydrocarbon molecules in the class of alkanes or aliphatic saturated hydrocarbons is determined in a similar way. In this article, we restrict ourselves to considering the structural device of alkanes.

Fig. 5 shows diagrams of the structures of simplest hydrocarbon molecules: methane (Fig. 5a) and ethane (Fig. 5b), in which the gravito-chemical bond is realized between paragravitational carbon atoms and hydrogen molecules ${ }^{1} \mathrm{H}_{2}$.
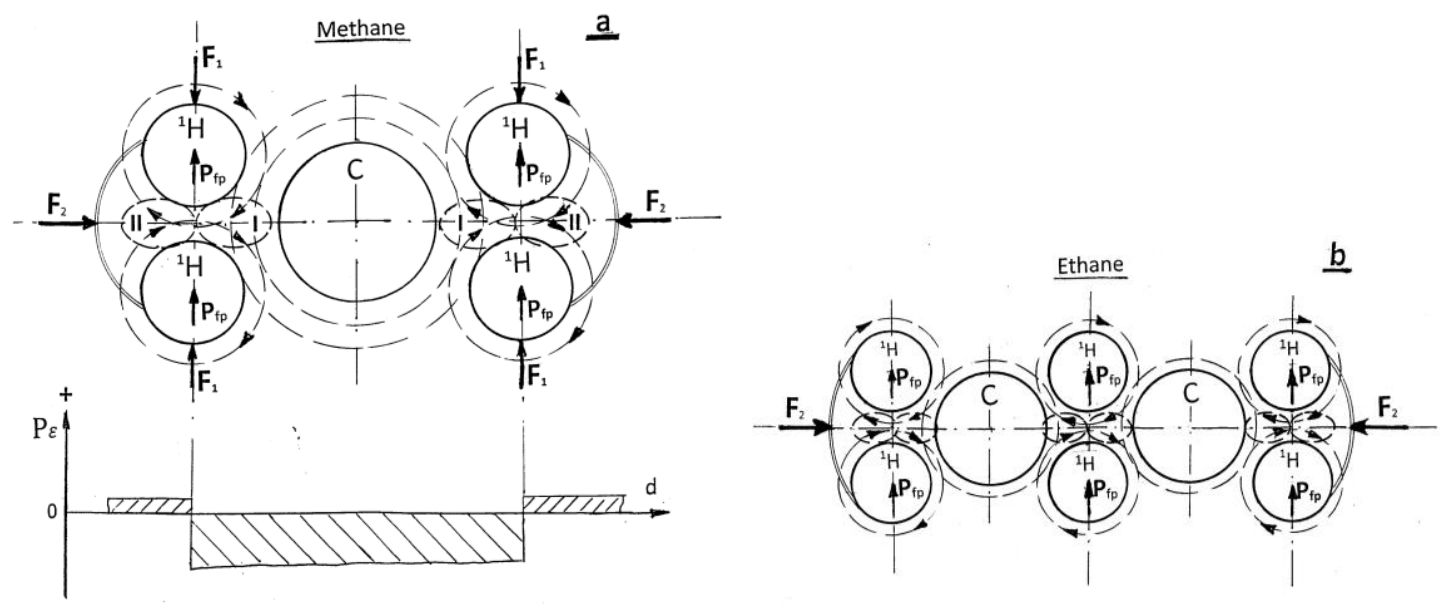

Fig. 5. Gravito-chemical bonding and structural device of methane and ethane molecules.

Shown in Fig. 5, as applied to the methane molecule, the distribution of pressures $\mathrm{P}_{\varepsilon}$ of "Dark energy", the forces of which $\mathbf{F}_{1}$ and $\mathbf{F}_{2}$ pressing the atoms, respectively, into the composition of the ${ }^{1} \mathrm{H}_{2}$ molecule and into the molecule of methane as a whole. The above forces of chemical bonding, which determine the formation of methane molecules are similarly realized in other hydrocarbons, for example, in ethane and propane.

According to the concept of the gravito-chemical bond which is realized in hydrocarbons in the framework of the paragravitational scenario described above, it is assumed that ${ }^{1} \mathrm{H}_{2}$ molecules, depending on their location in the molecules, can exhibit valence (with respect to hydrogen) equal 2 and 4 . The last circumstance should not scare anyone, since we are talking about valence, which refers to a complex of atoms in a hydrogen molecule, and not to an individual atom. Under these conditions, it is possible, for example, to take the PGF regions formed by the ${ }^{1} \mathrm{H}_{2}$ molecule as a conditional element of the chemical bond, assigning a valence of 2 to each of them.

As shown above in this article, the fundamental principle of the decomposition of molecules is reduced to the formation in the interatomic regions of the prevailing force of the Ferrogravitational field. The scheme of the influence of technical FGF on the methane molecule, leading to the decomposition of the molecule, is shown in the left part of Fig. 6. In this case, the BV of the technical FGF is directed perpendicular to the axis of the molecule what changes the Paragravitational state of the GF in the interatomic regions molecule ${ }^{1} \mathrm{H}_{2}$ on Ferrogravitational state. As for the right side of Fig. 6, then the initial state of the methane molecule is preserved here before the ferrogravitolysis process is switched on. 


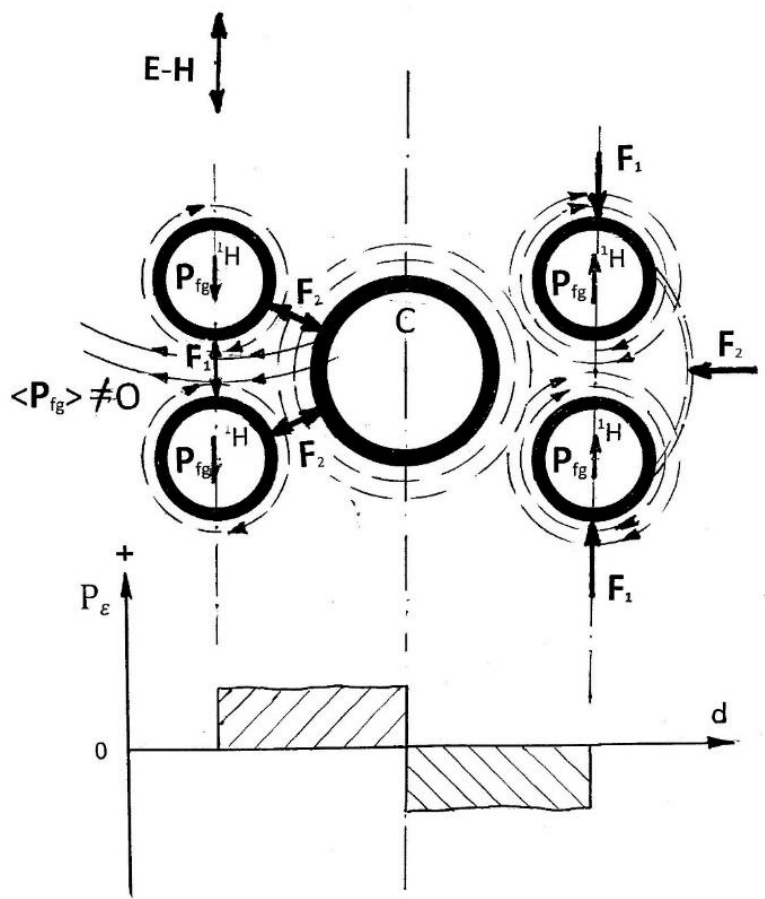

Fig. 6. The scheme of the ferrogravitolysis of methane molecule (see, the left part of Fig. 6).

As a result of the noted process, the decomposition of the hydrogen molecule in the system of the methane molecule is realized. In the subsequent process, after the destruction of the hydrogen molecule, the repulsion of hydrogen atoms from carbon occurs due to the same Gravitational levitation effect which is now realized between the hydrogen and carbon atoms. Thus, the process of ferrogravitolysis of a methane molecule is a two-step process and depends both on the strength of the technical FGF and on the orientation of the axis of the molecule relative to its $\mathbf{B V}$.

Recall that within the framework of the presented scheme of the effect of technical FGF on a methane molecule, similar to the conditions of magnetization, the ferropolarization vectors $\mathrm{GF}_{\mathrm{s}}$ of hydrogen atoms are directed along the $\mathbf{B V}$ of the technical field.

As for the technological scheme, in which the $\mathbf{B V}$ of the technical FGP is directed along the axis of the methane molecule, in this case, the violation of the PG-state of GF between the hydrogen atoms and the decomposition of its molecule does not occur.

Taking into account the peculiarities of the structure of hydrocarbon molecules (alkanes) presented in the article, it can be assumed that the described physics ferrogavitolises methane is characteristic of all representatives of this class.

\section{Structures and Ferrogravitolysis of Molecules Water}

The study by author of the structural states in the composition of water under the conditions of gravitochemical binding of hydrogen and oxygen atoms has shown that water is the heterogeneous mass in which have two stable molecular structures are realized, corresponding to the chemical formula $\mathrm{H}_{2} \mathrm{O}$ (shown in Fig. 7).

First of all, these are water molecules formed according to the hydrocarbon scenario in which the paragravitational oxygen atom binds to the ${ }^{1} \mathrm{H}_{2}$ molecules, that leads to the formation of so-called paragravitational molecules or PG-clusters (PGC) with the classical formula $\mathrm{H}_{2} \mathrm{O}$ (shown in Fig. 7a).

The second stable structural formation in the composition of water, corresponding to the classical chemical formula $\mathrm{H}_{2} \mathrm{O}$ (shown in Fig. 7b) is referred to as the ferrogravitational cluster (FGC). The point is that, unlike gravitationally stable carbon, oxygen is a gravitoplastic element. Under the influence of ferrogravitational fields of atoms of the nearest environment, in our case these are hydrogen atoms $\left({ }^{1} \mathrm{H}\right)$, the PGF of oxygen atom can be transformed into FGF. In this case, the formation of a gravitochemical bond between oxygen and hydrogen atoms ${ }^{1} \mathrm{H}$ will depend on the conditions of mutual orientation of the ferropolarization vectors $\mathbf{P}_{\mathrm{fp}}$ of the gravitational fields of atoms in the molecule in full accordance with the hydrogen bond conditions described in section 4.1 of the article.

The forces $\mathbf{F}$ responsible for the chemical bond in the molecules, as well as the ferropolarization vectors $\mathbf{P}_{\mathrm{fp}}$ of the gravitational fields of atoms in the FGC composition are shown in Fig. 7 b. Fig. 7 also shows diagrams corresponding to the pressure zones $\mathrm{P}_{\varepsilon}$ of "Dark energy", the forces of which are responsible for both the chemical bond in the ${ }^{1} \mathrm{H}_{2}$ molecule so and in the composition of the presented aqueous molecular clusters as a whole. 

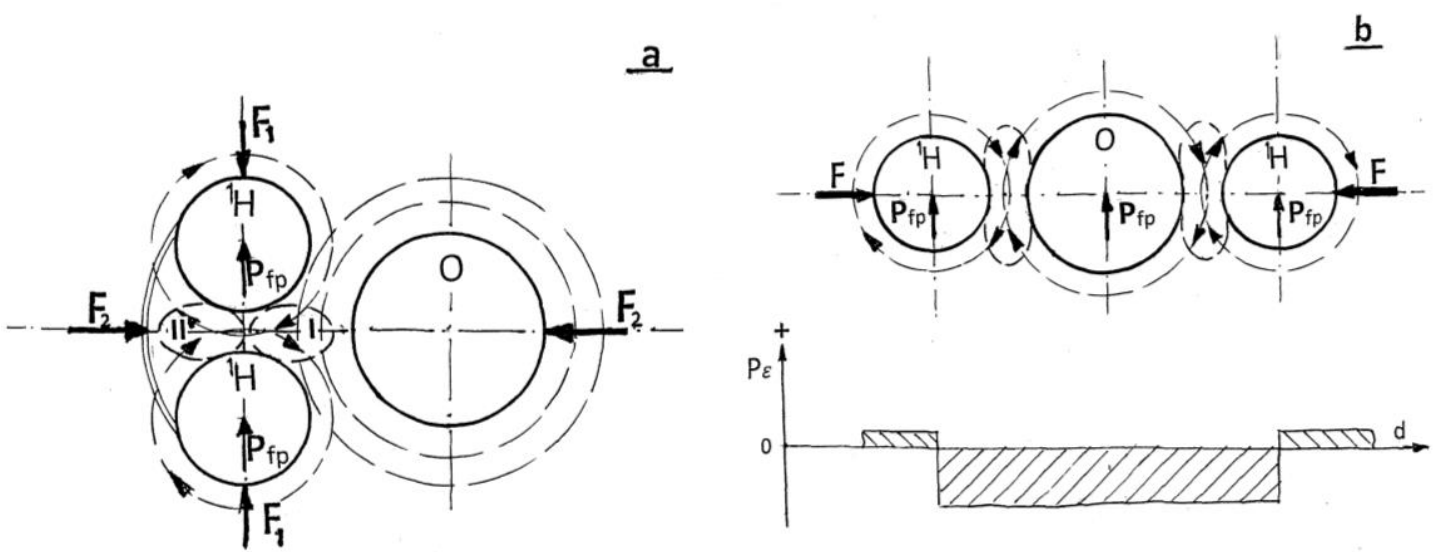

Fig. 7. The structural device and forces of gravito-chemical bonding in stable PGC (7a) and FGC (7b) clusters that make up the water mass.

The technological methods described above for the decomposition of hydrogen and hydrocarbon molecules, in full physical correspondence, are implemented in relation to the decomposition of the noted clusters in the composition of water.

Thus, the ferrogavitolisis of PGC is implemented in full accordance with the physics of decomposition of hydrocarbon molecule presented in the article the example of decomposition of a methane molecule and shown in Fig. 6. It is important to note that the complete analogy of noted decomposition PGC is determined by requirement of the paragravitational-state of GF of oxygen atom.

As for FGC, the gravito-chemical bond in which refers to the hydrogen bond described above, the technology of decomposition of these compounds occurs in accordance with the conditions of decomposition of hydrogen molecules, described in detail above in Section 4.1 (see Fig. 4b).

\section{Natural Ferrogravitolysis OF $\mathrm{H}_{2} \mathrm{O}$ and $\mathrm{CO}_{2}$ Molecules IN Leaf Cells of GReEn Plants AND ALGAE}

The ferrogravitolysis processes of water and hydrocarbon molecules presented in the article is based on the natural processes of decomposition of molecules, which are realized in the cells of leaves of green plants and algae by means of natural FGF. The existing biophysics considers the processes of decomposition of the molecules presented in the article as result of the action of photons on the cells of the leaves of green plants within the framework of a complex physicochemical process called photosynthesis. It is believed that it is the of photons, ultimately, are responsible for the decomposition of water and carbon dioxide molecules and provide nutrition for plants and algae.

The author's studies have shown that photons in the processes of photosynthesis they are only activators the processes of GF ferropolarization of chlorophyll emitted by cells in the leaves of green plants. The latter process is primarily determined by the polarization of the $\mathrm{FGF}_{\mathrm{s}}$ of ${ }^{1} \mathrm{H}$ hydrogen atoms, which, in chlorophyll, are found mainly in the $\mathrm{CH}_{2}$ and possibly $\mathrm{CH}_{3}$ hydrocarbon groups. In addition, hydrogen atoms in chlorophyll included in the atomic groups of $\mathrm{H}_{2} \mathrm{O}$, as well as in $\mathrm{OH}$ compounds.

It is the hydrogen atoms, which are stable sources of FGF, under the conditions of polarization of their gravitational fields in the composition of chlorophyll, that form the ferrogravitational field of plant cells, which decomposes water and $\mathrm{CO}_{2}$ molecules. As is known, both light and dark processes of photosynthesis are considered in biophysics. Such definitions, according to the author, relate exclusively to the degree of polarization $\mathrm{GF}_{\mathrm{s}}$ of protium atoms in the chlorophyll of plant cells.

So, for example, the state of polarization $\mathrm{GF}_{\mathrm{s}}$ of protium atoms in algal cells under normal conditions is sufficient to create the necessary FGF strength and implement the processes of ferrogravitolysis of water and carbon dioxide molecules without photonic action. As for the leaves of green plants, for the formation of the necessary FGF strength in cells and the implementation of the mechanism of decomposition of $\mathrm{CO}_{2}$ and water molecules, it is necessary to stimulate the processes of polarization of $\mathrm{FGF}_{\mathrm{s}}$ of protium atoms which is realized through photons.

It is not hard to understand that that the strength of FGF emitted by plant cells is most likely very low. Against this background, the process of ferrogravitolysis molecules $\mathrm{CO}_{2}$ and water in region with high strength of ferrogravitational field, generated by technical FG-generators, should occur almost instantaneously and carried out in the large flows of gas and water.

Here it is important to emphasize once again that in the optimal version, the technology we propose for the decomposition of $\mathrm{CO}_{2}$ and water molecules is carried out directly in the streams at the outlet of the source and does not require, for example, such complex and expensive operations as capturing and storing 
gases.

Technical FG-generators, creating high-intensity fields, are capable of replacing large forest areas in terms of the efficiency of $\mathrm{CO}_{2}$ and $\mathrm{CO}$ decomposition. In addition, such generators are capable of efficiently decomposing molecules in dirty effluents. In addition, such generators are capable of efficiently decomposing molecules in dirty effluents.

\section{TeChnicAl Generating OF FGF. FG - GenERATORS}

As a result of experimental and theoretical studies of magnetic charges extracted from the composition of substance the author has developed technology for enriching the magnetic charging of solids. The obtained by author the difference of the magnetostatic potential of magnetically charged bodies, allowed him to realize direct currents of magnetic charges in a superconductor. The final result of the noted works of the author was the experimental ferrogravitational field, which was obtained as a result of passing joint linear currents of electric $\mathbf{J}_{\mathrm{e}}$ and magnetic $\mathbf{J}_{\mathrm{g}}$ charges in a superconducting solenoid (see [2]-[4]).

The FGF is formed as a result of two successive processes: 1) $k_{1}\left(\mathbf{J}_{e}+\mathbf{J}_{g}\right)=\operatorname{rot}\left[\mathbf{J}_{e}-\mathbf{J}_{g}\right]$ and 2$) k_{2} \operatorname{rot}\left[\mathbf{J}_{e}-\right.$ $\left.\mathbf{J}_{\mathrm{g}}\right]=\operatorname{rot}[\mathbf{E}-\mathbf{H}]$.

In the first process, a vortex electromagnetic current is generated in a superconductor in the form of

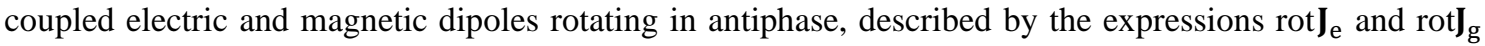
respectively. Within the framework of the second process, a gravitational field is formed around the bundle of two marked electric and magnetic dipoles $\operatorname{rot}\left[\mathbf{J}_{\mathrm{e}}-\mathbf{J}_{\mathrm{g}}\right]$ which is described by the vortex vector $\operatorname{rot}[\mathbf{E}-$ $\mathbf{H}]$. The considered above bunch of two dipoles (electric and magnetic) is an elementary source of the gravitational (ferrogravitational) field.

In the author's publications, this dipole bunch is referred to as $\mathrm{S}$ - Graviton, where $\mathrm{S}$ is from the word source. It is S - Gravitons, which should not be confused with Bohr's gravitons, that are the sources of all gravitational fields in Nature. S-Gravitons make practically all the electromagnetic density of shells atoms and nucleons, forming their gravitational fields.

The results of many years of research on magnetic charges and their currents, as well as his experiments with the generating of ferrogravitational field allowed the author to propose for practical implementation three design schemes for technical generating of ferrogravitational field.

1) solenoidal generator of FGF;

2) solenoidal generator of FGF with a gravito-soft core, i.e. the ferrogravity analogue of electromagnet;

3) permanent ferrogravitogenerator PFGG, i.e., the ferrogravity analogue of permanent magnet.

It should be noted that the most attractive, for wide practical use, are PFG-generators.

The detailed description of all these devices can be found in [20], [24].

\section{SCHEME OF THE INSTALLATION FOR FERROGRAVITOLYSIS OF WATER AND HYDROCARBONS}

A schematic diagram of plant for the decomposition hydrocarbons and $\mathrm{H}_{2} \mathrm{O}$ using FGF is shown in Fig. 8. The flows of water or gases, in this case of hydrocarbons, through the pipe 1 is fed to the FGF oblast which is formed by the FG-generator 2. In the optimal variant, such a generator should be the PFGG. However, this field, as shown by the author's experiments, may well be created by means of a solenoidal generator.

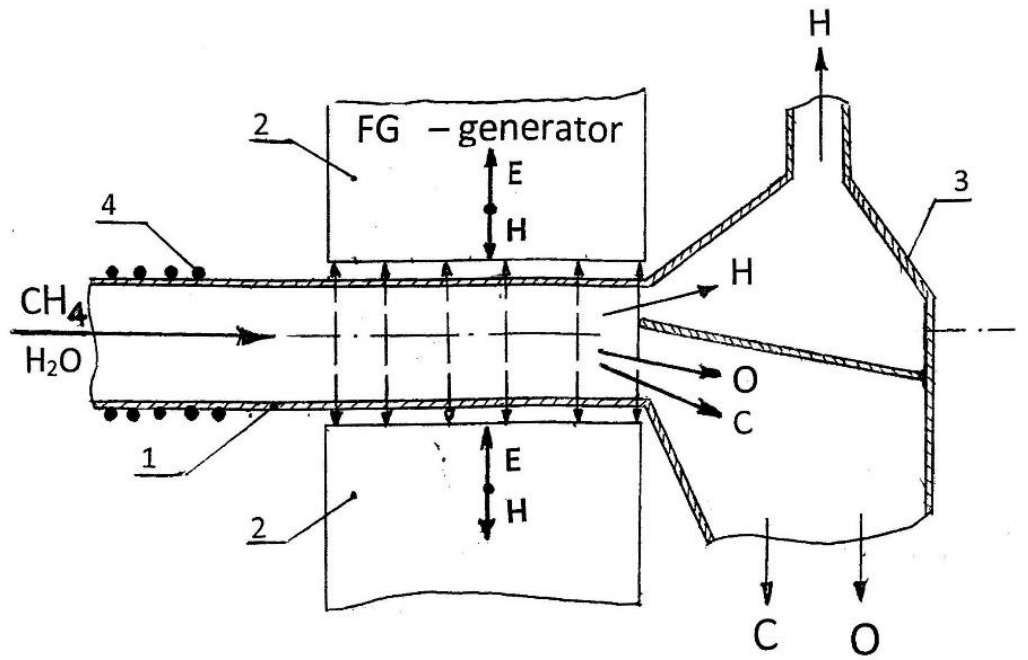

Fig. 8. Schematic of a plant for the ferrogravitolisis of water and hydrocarbons. 
At the exit from the FGF zone (molecular decomposition zone), the fluxes of hydrogen, oxygen and carbon atoms after decomposition are directed to a separator (3), in which they are finally separated using differences in their gravitational properties. At the entrance to pipe 1, a heater (4) is installed, which is designed to heat molecules in streams, which, according to the author, can stimulate the efficiency of the subsequent disintegration of molecules.

\section{CONCLUSION}

The discovery and study of real magnetic charges, as well as true antielectrons in the compositions of atoms and substance allowed the author to develop and propose to the scientific community the foundations of general physics with the participation of these real particles, which almost 150 years, have been ignored in physical science. Physics with real magnetic charges according to the author is an inevitable replacement for the existing vicious electronic physics [26].

The introduction of real magnetic charges, as well as true anti-electrons in fundamental physics, radically changes, practically, all existing physical and physicochemical concepts. So, for example, the gravitational field, in physics with real magnetic charges, is just a vortex electromagnetic field, which is described by the vortex vector $\operatorname{rot}[\mathbf{E}-\mathbf{H}]$. It was the elucidation of the real physics of the gravitational field that allowed the author to develop and propose a concept of chemical bonding that actually works in nature and which is based on the gravito-chemical bond.

The vicious situation in physics described in the article, associated with ignoring magnetic charges and true antielectrons, was the main reason for the emergence of such well-known theoretical "discoveries" as the global expansion of the Universe, a very big bang, annihilation of the particle-antiparticle, field and Higgs boson as the root cause of formation particles' masses, representation of the gravitational field in the form of a curved four-dimensional space-time and many others.

Paradoxically, vicious electronic physics turned even such fundamental physical concepts as Matter and Antimatter into the category of false ideas. As noted above, in physics with real magnetic charges, the concept of Matter or Material phase refers exclusively to fundamental electric and magnetic particles with a negative charge. The electron is the representative of Matter. As for the positively charged fundamental particles, both electrical and magnetic, they constitute Antimatter or Antimater phase. Therefore, atoms, nucleons, substance are not Matter at all. All these last electromagnetic formations are Physical Masses, consisting of material and antimaterial particles (see [5]-[7]). The above correction of fundamental concepts also assumes that Antimatter and Antisubstance are completely different physical categories.

Note 6. However, as it usually happens in life, any delusion, even such a grandiose like what happened in physics and described above, sooner or later passes away. Therefore, in the future we still have a very "fascinating" spectacle with the "fall" of the modern (electronic) physical theory from the conquered "peaks". At the same time, the "thunder" from such fall will be the stronger the longer in time pull back the inevitable processes of physics correction are implemented. Hence only those who can live up to this moment will be able to see this.

\section{ACKNOWLEDGMENTS}

The author expresses deep gratitude to Elena Sizova and Vitaly Sizov who for many years helped and supported him which largely contributed to the implementation of his research activities.

\section{APPENDIX}

\section{1) The main reasons for vicious ignoring real magnetic charges in existing physical science}

Real magnetic charges, as well as true antielectrons, which make up 3/4 of atomic shells, were "buried alive" in existing physics as a result of four basic physical and general-human circumstances (see [5-7]).

2) The physics of rigid retention of magnetic charges in a substance, which is fundamentally different from the retention of electrons

It is the physics of rigid confinement, magnetic charges and true antielectrons in matter, which is fundamentally different from the retention of electrons, that is the main reason why these particles are very difficult to experimentally isolate from the composition of matter and thoroughly investigate. However, the difficulties of experimental work with magnetic charges in substunce do not mean that these particles should have been ignored. It should also be noted that the retention forces of the noted problem particles in the substance increase with an increase in its internal energy. The latter circumstance explains the reasons for unsuccessful attempts to detect these particles in high-energy experiments (see, [8]). 
3) The primitive-superficial perception of J. Maxwell in 1873 of the results of well-known Oersted experiment.

It is precisely J. Maxwell, based on his primitive and superficial impression of the famous Oersted experiment, introduced into physics the vicious myth that the direct sources of the magnetic field are moving electrons. The so-called first Maxwell equation $\mathrm{k} \mathbf{J}_{\mathrm{e}}=\operatorname{rot} \mathbf{H}$, in fact, is erroneous, since it involves two different physical processes: 1) $\mathrm{k}_{1} \mathbf{J}$ e $=\operatorname{rot} \mathbf{J}_{\mathrm{g}}$ and 2) $\mathrm{k}_{2} \operatorname{rot} \mathbf{J}_{\mathrm{g}}=\operatorname{rot} \mathbf{H}$, where $\operatorname{rot} \mathbf{J}_{\mathrm{g}}$ is the vortex orbital current of magnetic charges (a rotating magnetic dipole). The fact is that electrons in the composition of an electric current are exclusively mediators, which are only capable of spinning magnetic dipoles that actually exist in substance (see the first equation). As for the magnetic field, its direct sources are real magnetic charges which, in the compositions of rotating magnetic dipoles, form the well-known vortex magnetic field rotH (see, the second equation and [10], [14]).

4) The inertia of erroneous human thinking.

During almost 150 years of the dominance of Maxwell's vicious electric magnetism, theoretical "Himalayas" were built in physical science, on which, starting from "kindergarten", many generations of scientists were brought up. The antagonism to real magnetic charges and true anti-electrons in the physical community is so strong that on all the so-called serious information levels, as they say, from the threshold, any movements of thought and even experimental results, associated with the marked problematic particles.

\section{5) The discriminatory policy of the leading physical publications.}

For example, the articles of author with the results of the study of real magnetic charges, sent to the socalled prestigious journals (Phys.Rev, Nature, etc.) were immediately repudiate, with expert opinions which, in general, can be described by the expression: this can never be. It is the leading physics publications they stand guard over the existing theoretical physics, creating numerous "chippers" that prevent the penetration of views on their pages that are alien to official physical dogmas.

Of course, it is possible to understand the noted "defensive" reaction of the Editorials and their experts, since the introduction into fundamental physics of real magnetic charges, as well as true antielectrons, presupposes a radical revision of the entire theoretical arsenal of physics created in 150 years of ignoring these particles. By the way, this arsenal accounts for at least $70 \%$ of the content of the leading physical journals for all the years of the dominance of vicious electric magnetism in the sciences, i.e. counting from 1873.

\section{REFERENCES}

[1] Robert A. Sizov. The Experiments Detecting of Real Magnetic Charges in Structures of Atoms and Substance. Journal of Modern Physics, 2020; 11:1245-1261. https://doi.org/10.4236/jmp.2020.118078.

[2] Robert A. Sizov. Experimental Ferrogravitational Field around Untwisting Closed Superconductor. Journal of Modern Physics, 2020; 11:1807-1826. DOI: 10.4236/jmp.2020.1111113.

[3] Robert A. Sizov. The magneto-motive force, currents of magnetic and electric charges in static superconductor and them vortex spinor fields. Journal Current Trend in Physics Research and Applications, 2021; 2(1): 1-10. https://katalystpub.com/jctpraarticles-inpress/.

[4] Robert A. Sizov. Real magnetic poles (magnetic charges) in the Physics of magnetism, gravitation and levitation. Journal of Modern Physics, 2015; 6:1013-1022. http://dx.doi.org/10.4236/jmp.2015.68106.

[5] R.A. Sizov. New Presentation of Nature Magnetism, Gravitation and Nuclear Forces of Bonding. Akademizdat Center "Science", Moscow, 62 pages, 2001.

[6] Robert A. Sizov. Electromagnetic Shells of Atoms and the Periodic System of Elements. Journal of Modern Physics, 2016; 7:2374-2397. http://dx.doi.org/10.4236/jmp.2016.716206.

[7] Robert A. Sizov. Magnetic and Electric Charges in Physics and Technology of Electromagnetic and Magnetoelectric Induction. Journal of Modern Physics, 2017; 8:964-981. DOI: 10.4236/jmp.2017.86061.

[8] Robert A. Sizov. "Electrical" and "Magnetic" Worlds in Universe. Journal of Modern Physics, 2017; 8:1072-1086. DOI: 10.4236/jmp.2017.87069.

[9] J.C. Maxwell. Treatise on Electricity and Magnetism. 1873, Vol. 1-2 Clarendon Press, Oxford.

[10] R.A. Sizov. The electrical magnetism of Maxwell (1873) is the "crooked mirror" of physical science. Applied Physics Research, 2019; 11(3):49-55. http://dx.doi.org/10.5539/apr.v11n3p49.

[11] P.A.M. Dirac. Quantized Singularities in the Electromagnetic Field. Proc. Roy. Soc. A133, 60-72, 1931. http://dx.doi.org/10.1098/rspa.1931.0130.

[12] R.A. Sizov. Real Magnetic Charges as A Negation of the Electric Magnetism Maxwell and Electrified Dirac's Monopole. Phys. Astron. Int J. 2018; 2(1): 00043. DOI: 10.15406/paij.2018.02.00043.

[13] S. T. Bramwell S. R. Giblin S. Calder, R. Aldus, D. Prabhakaran, T. Fennell. Measurement of the charge and current of magnetic monopoles in spin ice. Nature, 2009; 461:956-959. doi:10.1038/nature08500.

[14] Robert A. Sizov. Great Mistake of Great J. C. Maxwell. Applied Science and Innovative Research, ISSN 2474-4972 (Print) ISSN 2474-4980 (Online). 2020; 4(1). doi:10.22158/asir.v4n1p32.

[15] F. Ehrenhaft. On Photophoresis, the True Magnetic Charge and on Helical Motion of Matter in Fields. Acta Physica Austria, $1952 ; 5: 12-29$

[16] V. F. Mikhailov and J. Ruzicka. Magnetic charge in the experiments by F. Ehrenhaft and their modern development, Acta physica Univ. Comen., XXIX, pp. 97-148, 1989.

[17] R.A. Sizov. Matter, Antimatter and Energo-Medium are Physical Triad of the Real World. Akademizdat-Center "Science", Moscow, 192 p. 2012.

[18] Robert A. Sizov. World Physical Triad: Matter, Antimatter and "Dark Energy" in the Processes of Climatic Changes on the Earth. Journal of Modern Physics, 2016; 7:558-572. http://dx.doi.org/ 10.4236/jmp.2016.76059. 
[19] Robert A. Sizov. Nuclear Physics with Magnetic Charges. Journal of Modern Physics, 2018; 9(2):145-171. DOI: 10.4236/jmp.2018.92010.

[20] Robert A. Sizov. Real Magnetic Charges in the Substance, Ferrogravitation and Technical Levitation. Journal of Modern Physics, 2015; 6:1591-1601. http://dx.doi.org/10.4236/jmp.2015.611161.

[21] Robert A. Sizo. Gravitational Chemical Bond with Real Magnetic Charges and True Antielectrons. Open Journal of Physical Chemistry, 2020; 10: 48-67. DOI: 10.4236/ojpc.2020.101003.

[22] R.A. Sizov. Dirac's "holes" are the true Antielectrons and real particles of Antimatter. Journal of Modern Physics, 2015; 6 (2015):2280-2289. http://dx.doi.org/10.4236/jmp.2015.615232.

[23] Robert A. Sizov. Law of universal gravitation with real magnetic charges and "dark energy". Phys. Astron. Int. J., 2018;2(5):439443. DOI: 10.15406/paij.2018.02.00121.

[24] Robert A. Sizov. Decomposition $\mathrm{CO}_{2}$ and $\mathrm{CO}$ in Flow of Gases by Means of Technical Ferrogravitational Field. European Journal of Applied Physics, 2021; 3(3), 21-28. https://doi.org/10.24018/ejphysics.2021.3.3.78.

[25] Robert A. Sizov. The Gravito-Chemical Bond and Structures of Hydrocarbons and Water Molecules with Real Magnetic Charges. Open Journal of Physical Chemistry, 2021; 11(4), September 30, 2021. DOI: 10.4236/ojpc.2021.114013.

[26] Robert A. Sizov. The "Naked Electron". Applied Science and Innovative Research ISSN 2474-4972 (Print) ISSN 2474-4980 (Online), 2019; 3(4). http://dx.doi.org/10.22158/asir.v3n4p257.

[27] Robert A. Sizov. "Particle of God" as insulting of the Divine Principle. International Journal of Theoretical \& Computational Physics, 2021. doi.org/10.47485/2767-3901.1010.

Robert A. Sizov is Doctor of Physical and Mathematical Sciences, specialist in the oblast of general physics with real magnetic charges. Currently, he works as an individual researcher. Extended information about his research on the topic: real magnetic charges in atoms and substance can be found by typing in Google: Robert Sizov Physics. 